analysis of variance, $\mathrm{p}<0.05)$.

Conclusion: The spatial resolution revealed an EM gradient within Met but not Pri. This provides an unprecedented view of the EM gradient during the progression of cancer such as OCCC. Our study provides the first spatially resolved in situ evidence of intermediate or hybrid EM states within the tumor samples of similar morphology.

Poster (008)

Epithelial Ovarian Cancer including Borderline Tumor

https://doi.org/10.3802/jgo.2021.32.\$1.008

\section{DKK3, down-regulated in invasive epithelial ovarian cancer was associated with chemoresistance and enhanced paclitaxel susceptibility via inhibition of $\beta$-catenin-P- glycoprotein pathway}

\author{
Que Thanh Thanh Nguyen, ${ }^{1}$ Kyung-Mi Choi, ${ }^{2}$ Tae Jin Lee, ${ }^{3}$ \\ So Young Kim, ${ }^{2}$ Jae Hyung Kim, ${ }^{4}$ Eun-Ju Lee, ${ }^{2, *}$ \\ 'Chung-Ang University, Seoul, Korea \\ ${ }^{2}$ Department of Obstetrics and Gynecology, Chung-Ang University School \\ of Medicine, Seoul, Korea (ejlee@cau.ac.kr) \\ ${ }^{3}$ Department of Pathology, Chung-Ang University School of Medicine, \\ Seoul, Korea \\ ${ }^{4}$ Department of Radiology, Sanggye Paik Hospital, Inje University College of \\ Medicine, Seoul, Korea
}

Objective: This study aimed to assess aberrant Dickkopf-3 (DKK3) expression in epithelial ovarian carcinoma and to determine the therapeutic role of DKK3 in ovarian cancer. Methods: DKK3 protein expression was examined by immunohistochemistry using tissue blocks from 82 patients with invasive ovarian adenocarcinoma, and 15 normal, 19 benign, and 10 borderline tumors as controls. Survival data were estimated using Kaplan-Meier estimates, and multivariate analysis was performed using the Cox regression method. Paclitaxel-resistant cell lines were manufactured with TOV-21G and OV-90 ovarian cancer cell lines. The protein expression and cell viability were assessed by Western blotting and MTT assay, respectively.

Results: DKK3 was significantly down-regulated in invasive carcinoma compared to normal, benign, and borderline tumors. Univariate analysis showed that higher FIGO stage, DKK3 loss, suboptimal debulking operation, and chemo-resistance were significantly associated with low disease-free survival of serous carcinoma patients. DKK3 loss occurred in $56.1 \%$ invasive carcinoma cases and was significantly associated with chemoresistance in serous adeno carcinoma $(\mathrm{p}=0.029)$. Paclitaxelresistant cell lines were successfully established. Western blot analysis showed that DKK3 was lost in paclitaxel-resistant cells and as expected, $\beta$-catenin and P-glycoprotein were upregulated. Secreted DKK3 exerted an anti-proliferative effect and induced paclitaxel susceptibility in paclitaxel-resistant cells $(\mathrm{p}<0.01)$. Secreted DKK3 was internalized into cells, reduced $\beta$-catenin activity, hence inhibited P-glycoprotein, suggesting an axis link between them.

Conclusion: DKK3 loss was frequent in invasive epithelial ovarian cancer and significantly associated with chemoresistance. Secreted DKK3 has an anti-proliferative effect on paclitaxel-resistant cells by inhibiting $\beta$-catenin and P-glycoprotein, suggesting that DKK3 could be therapeutics targeting paclitaxel-resistant ovarian cancer.

Poster (009)

Conservative \& Fertility Preservation

https://doi.org/10.3802/jgo.2021.32.\$1.009

\section{Fertility sparing surgery in malignant ovarian germ cell tumors (MOGCTs): 15 years experiences}

\author{
Shima Alizadeh, " Narges Zamani, Mohadese Rezaei Poor, \\ Sedigheh Ghasemian Dizajmehr, Mitra Modares Gilani \\ Tehran University of Medical Sciences, Tehran, Iran \\ (alizadeh.shima@gmail.com)
}

Objective: Our aim is to evaluate the reproductive outcome of fertility-sparing surgery and chemotherapy among young women diagnosed with malignant ovarian germ cell tumor (MOGCT) of any stage.

Methods: In current retrospective study we evaluated 79 patients with MOGCT whom visited at Imam Center, Vali-Asr Hospital, Gynecology Oncology department during 2001-2016. Reproductive outcomes (menstruation status \& childbearing) followed fertility preserving surgery and adjuvant chemotherapy by filling questionnaires. Statistical analysis was done with SPSS software, $\chi^{2}$ tests were done, and significance determined at $\mathrm{p} \leq 0.05$.

Results: Among 79 young women who underwent fertilitysparing treatment,72 patients followed up for reproductive outcome and 7 patients excluded due to death ( 3 cases), XY genotyping ( 3 cases), and bilateral ovarian involvement (1 case). The mean age at presentation was 23 years (range $=19-33$ years). The 5 and 10-year disease-free survival rate was $87 \%$ and $94.4 \%$, respectively. The overall survival rate was $94.4 \%$ at 5 and 10 years. Regular menstruation recovered in 60 of 72 patients after treatment $(83 \%)$. All patients without adjuvant chemotherapy experienced regular menstruation while normal menstruation retrieved in $78 \%$ in adjuvant chemotherapy group at the end of treatment. This retrieval of regular menstruation was not 